\title{
CULTURA POLÍTICA, INSTITUIÇÕES E EXPERIÊNCIA DEMOCRÁTICA NO BRASIL
}

\author{
Ednaldo Aparecido Ribeiro
}

\begin{abstract}
RESUMO
O artigo pretende, por meio da análise de dados produzidos pelo projeto World Values Surveys, identificar o impacto que o período de vigência de instituições democráticas em nosso país produziu sobre as faixas etárias mais jovens da população, que tiveram suas experiências de socialização política sob tal forma de governo. Partindo do pressuposto de que a incorporação de valores políticos ocorre, principalmente, nas duas primeiras décadas de vida do indivíduo, procuramos verificar a validade da hipótese de que as instituições democráticas tenderiam a favorecer o estabelecimento de valores congruentes e favoráveis à sua consolidação e aprofundamento. Para tanto, foram empregados testes de associação entre a variável idade e algumas medidas de valores e atitudes políticas enfatizadas por diferentes abordagens da chamada "teoria da cultura política". Os resultados obtidos contrariam a tese mencionada, ao menos para o caso brasileiro, uma vez que não foram identificados valores e atitudes significativamente diferentes entre faixas etárias que tiveram seu processo de socialização política sob diferentes arranjos institucionais.
\end{abstract}

PALAVRAS-CHAVE: instituições democráticas; valores políticos; socialização política; faixas etárias.

\section{I.INTRODUÇÃO}

Nas últimas décadas um considerável grupo de sociólogos e cientistas políticos tem afirmado a importância de variáveis culturais para a explicação do surgimento, consolidação e aprofundamento de sistemas políticos democrátiCos (PUTNAM, 1996; DALTON, 2000; INGLEHART， 2001; NORRIS， 2002; INGLEHART \& WELZEL, 2005). Apesar de divididos em relação a quais variáveis teriam mais impacto e quanto aos fatores que conduzem à formação de valores e crenças compatíveis com a democracia, é consensual entre esses investigadores a afirmação de que a estabilidade desta forma de governo passa, necessariamente, pela internalização de um conjunto de normas e práticas democráticas compatíveis pela população.

Gabriel Almond e Sidney Verba (1989), no clássico The Civic Culture, trataram dessa relação entre orientações subjetivas e instituições a partir da tese da congruência, que pode ser sintetizada na afirmação de que o sucesso de um sistema político depende significativamente da compatibilidade entre suas instituições e conjunto de valores, crenças e atitudes partilhadas pela população.

A questão, entretanto, é bastante controversa, pois um grupo também expressivo de pesquisadores tem defendido que os processos de demo- cratização devem ser explicados por análises que enfatizem a atuação política das elites ou o funcionamento das instituições (O’DONNELL \& SCHMITTER, 1986; KARL \& SCHMITTER, 1991; PRZEWORSKI, 1992).

A discussão torna-se ainda mais intensa quando o foco de atenção concentra-se sobre a identificação da causalidade entre os fatores culturais e institucionais. De um lado, teríamos os adeptos de abordagens culturalistas, afirmando que uma configuração de elementos culturais compatíveis com a democracia precede a implantação das suas respectivas instituições. Por outro, encontramos os defensores da tese de que tais valores só podem desenvolver-se sob a existência de instituições democráticas, pois seria a experiência de vida nessas condições que conduziria à formação de mentalidades democráticas (MULLER \& SELIGSON, 1994).

Sem a pretensão de produzir respostas conclusivas sobre o tema, a intenção deste trabalho é contribuir minimamente para esta discussão, por meio da análise de algumas variáveis relacionadas à cultura política da população brasileira, tratando de identificar a existência ou não de variação em decorrência da faixa etária dos indivíduos. Levando em conta que o início do processo de estabelecimento de instituições democráticas no Brasil ocor- 
reu, aproximadamente, há duas décadas, pretendemos identificar se existe variação nos valores, crenças e atitudes políticas entre grupos etários distintos, submetidos a diferentes experiências políticas, vivendo sob diferentes modelos institucionais.

Assumindo a tese proposta pelos estudiosos do processo de socialização de que os valores políticos são predominantemente formados nas duas primeiras décadas de vida (ROKEACH, 1968; ALMOND \& POWELL JR., 1972; INGLEHART, 2001), caso a experiência de viver sob instituições democráticas conduzisse à formação de valores pró-democracia, deveríamos encontrar entre os membros de grupos etários jovens, socializados na vigência de tal arranjo institucional, uma postura relativamente mais democrática. É sobre esta hipótese que pretendemos nos debruçar.

Ainda que não constitua-se um teste definitivo, uma vez que, para tal, seria necessária uma análise longitudinal envolvendo um período maior de tempo, acreditamos que o exercício seja interessante para refletirmos sobre os limites e desafios que colocam-se diante da consolidação e do aprofundamento da jovem democracia brasileira.

Para alcançar nosso objetivo, organizamos o trabalho da seguinte forma: primeiramente, apresentamos algumas considerações teóricas relevantes sobre a relação entre cultura política e instituições, com atenção especial à polêmica sobre a causalidade entre estas duas dimensões; em seguida, passamos à apresentação de nossa proposta de análise acerca da influência da recente experiência democrática nacional sobre os valores da população, dividida em grupos etários, portadores de experiências distintas de socialização política.

\section{CULTURA POLÍTICA E INSTITUIÇÕES DEMOCRÁTICAS}

Apesar de a noção de cultura política ter uma história longa, a primeira tentativa de formulação sistemática de um programa de pesquisas tendo os valores políticos como objeto central data da década de 1960. The Civic Culture, de G. Almond e S. Verba, inaugura esse programa, trazendo a tese fundamental de que os valores, conhecimentos e crenças políticas dos indivíduos são fundamentais para a configuração de qualquer sistema político (ALMOND \& VERBA, 1989).
Esta afirmação básica foi o ponto de partida dos autores para a realização de um estudo que estendeu-se por cinco países (Estados Unidos, Inglaterra, Itália, Alemanha e México), com o objetivo de investigar o grau de congruência entre este conjunto de variáveis subjetivas e o sistema político.

Diante de tal empreendimento, definiram cultura política como "[...] the particular distribution of patterns of orientation toward political objects among the members of the nation [...]" (ALMOND \& VERBA, 1989), ou seja, um conjunto de orientações políticas subjetivas que poderia explicar a motivação subjacente às ações praticadas pelos atores, tendo como referência os objetos políticos, isto é, a totalidade do sistema político, envolvendo as estruturas de incorporação (inputs) e satisfação (outputs) de demandas individuais e coletivas, assim como o próprio indivíduo em seus papéis e atribuições políticas (idem, p. 14).

Como ocorre em todo empreendimento inovador, esta pesquisa inicial foi alvo de inúmeras críticas pertinentes, em especial, pelo seu demasiado “etnocentrismo' na definição do que deveria ser considerado como "político” (INGLEHART, 1988). Os autores teriam tomado o modelo anglosaxão de democracia liberal como o que teria as condições necessárias para o surgimento do modelo de cultura cívica. Ao definirem as características subjetivas de cada um dos modelos de cultura política, teriam colocado em posição confortável países como Estados Unidos e Inglaterra e condenado países como Alemanha e Itália a uma situação política desfavorável (RENNÓ, 1998).

Apesar dos problemas apontados, a proposta deste trabalho inaugural expressa a emergência de uma preocupação com a incorporação na análise política moderna de elementos que não referemse ao chamado "núcleo duro" da política. Assim, o seu mérito está em assumir o papel de connecting link entre o nível das atitudes e motivações subjetivas individuais e o sistema político em suas diferentes partes, tendo como principal preocupação a identificação de congruências ou incongruências entre estas duas esferas.

Após um momento de reflexão crítica e rompendo com alguns limites iniciais, os estudos nesta área foram continuados por uma série de pesquisadores espalhados por diferentes países e, atualmente, passam por um momento de intensa pro- 
dução (KLINGEMANN \& FUCHS, 1995; PUTNAM, 1996; PHARR \& PUTNAM, 2000; INGLEHART, 2001).

Esta retomada tem ocorrido também no Brasil, onde vários pesquisadores têm dedicado-se a investigações sobre o caráter da cultura política nacional, relacionando-a sempre com a possibilidade de fortalecimento do regime democrático recentemente implantado no país (LAMOUNIER, 1991; SALES, 1994; MOISÉS, 1995; CARVALHO, 1999).

Ainda que a concordância em relação à tese da congruência entre valores e instituições reúna tais pesquisadores nacionais e internacionais no grande grupo dos estudiosos da cultura política, diferenças internas significativas precisam ser destacadas.

Divergências importantes aparecem, sobretudo, quando trata-se de identificar quais valores, crenças ou atitudes teriam mais impacto sobre o sistema político. Destacando estas divergências, Inglehart e Welzel (2005) identificam, pelo menos, três abordagens distintas.

Primeiramente, temos uma corrente que enfatiza o tema da legitimidade ou do suporte à democracia (MISHLER \& ROSE, 2001; SELIGSON, 2002). A confiança nas instituições é tomada como uma variável fundamental para o funcionamento efetivo desta forma de governo por um conjunto expressivo de pesquisadores adeptos desta abordagem. A identificada tendência de declínio nos níveis de confiança em relação às instituições democráticas tem sido interpretada como algo preocupante, pois poderia resultar em uma crise de legitimidade (NEWTON \& NORRIS, 2000).

Um segundo grupo, que podemos classificar como comunitarista, extrapola os limites dos valores ligados às instituições e focaliza sua atenção sobre aqueles ligados à vida comunitária cotidiana dos indivíduos. Valores, crenças e atitudes que fortalecem os compromissos sociais e a lealdade dos cidadãos com a sua comunidade teriam mais poder explicativo sobre a democracia do que a confiança institucional (PUTNAM, 1996; 2000). Nesta perspectiva, a participação voluntária em associações e a confiança interpessoal seriam as variáveis mais relevantes para explicar a dinâmica institucional democrática.

Por fim, teríamos a abordagem do desenvol- vimento humano, que enfatiza os valores de autoexpressão como aqueles que realmente conduziriam ao estabelecimento e ao fortalecimento de democracias (INGLEHART, 2001; INGLEHART \& WELZEL, 2005). A disseminação de valores que conduzem à aspiração por mais liberdade, à luta pelo estabelecimento ou aprofundamento dos direitos civis e políticos teria uma grande capacidade explicativa sobre o processo de democratização, de acordo com os partidários desta corrente.

Essa última abordagem é particularmente interessante pelo que apresenta de evidências em relação à polêmica questão da causalidade entre valores e instituições, por isso, merece mais atenção de nossa parte.

A teoria do desenvolvimento humano pode ser inicialmente compreendida como uma estratégia de conciliação entre duas perspectivas aparentemente opostas no interior dos estudos sobre modernização e desenvolvimento político-econômico.

Inaugurada por Max Weber (1996), em seu clássico A ética protestante e o espírito do capitalismo, podemos identificar uma explicação que afirma a relevância de configurações culturais nos processos de desenvolvimento econômico e político. Na célebre análise weberiana, um conjunto de princípios religiosos que compunham uma determinada ética protestante é apontado como fator relevante para o estabelecimento de um ethos econômico-capitalista.

Recentemente, vários autores uniram-se a esta tese, como Fukuyama (1995), Huntington (1997) e Putnam (1996). Este último, em publicação bastante influente nas pesquisas sobre capital social, trata de demonstrar o quanto variáveis culturais podem determinar o sucesso político e econômico.

Em oposição, teríamos outro grupo de estudiosos defendendo a tese de que o processo de modernização seria acompanhado de um conjunto de mudanças culturais congruentes. Nesta segunda perspectiva, estariam agrupados autores clássicos como Karl Marx, com os conceitos de infra-estrutura e superestrutura e estudiosos mais recentes como Daniel Bell (1977), com sua tese acerca do advento da sociedade pós-industrial.

A proposta de Inglehart (2002) caminha no sentido de validar ambas as perspectivas. Por um 
lado, afirma existirem evidências suficientes para associar o desenvolvimento econômico com mudanças nos sistemas de normas e valores sociais. Tais mudanças caracterizariam-se pela substituição de valores tradicionais por outros modernos e pós-modernos, enfatizadores da qualidade de vida, da proteção ao meio ambiente e da auto-expressão (INGLEHART, 2000). De outro lado, também destaca que esta mudança segue caminhos distintos em cada nação, em decorrência de uma matriz cultural persistente construída ao longo da história. A mudança nos valores, desta maneira, manifestar-se-ia de diferentes maneiras em diferentes tradições culturais.

Aceitando a tese da divisão do globo em diferentes zonas culturais, como proposta por Huntington (1997), o autor afirma que diferentes tradições culturais influenciam de maneiras distintas o comportamento econômico e também político dos indivíduos. As zonas culturais teriam sido formadas por tradições que, mesmo diante dos processos de modernização, manifestam grande poder de permanência e influencia. Assim, “[...] o fato de que uma sociedade é historicamente protestante, ortodoxa, islâmica ou confuciana dá origem a zonas culturais com valores culturais muito distintos, que persistem quando isolamos os efeitos do desenvolvimento econômico" (INGLEHART, 2002, p. 135).

A solução conciliadora entre as duas posições apresentadas pode ser sintetizada na tese de que a modernização e o desenvolvimento econômico são acompanhados por uma mudança significativa nos valores sociais, porém, esta mudança processase sob caminhos relativamente estabelecidos por tradições culturais específicas, formadas ao longo da história de cada país e região, com alto poder de permanência.

Essa proposição é corroborada em obras como Modernization and Postmodernization: Cultural, Economic and Political Change in Forty-Three Societies (INGLEHART, 2001) e Modernization, Cultural Change, and Democracy: The Human Development Sequence (INGLEHART \& WELZEL, 2005), que apresentam análises de dados coletados pelo projeto World Values Survey ao longo de décadas, envolvendo um número crescente de sociedades.

Nestes estudos, os autores identificam uma série de diferenças significativas nas normas, va- lores e crenças políticas, sociais e religiosas das populações de nações ricas e pobres, bem como entre nações que compõem diferentes tradições ou zonas culturais.

Essa diferença apresenta-se de maneira significativa, principalmente, quando são isoladas duas dimensões deste conjunto de variáveis culturais: as orientações secular-racionais e os valores de auto-expressão. A partir destes dois eixos fundamentais, o autor propõe uma localização para cada sociedade dentro de um mapa cultural mundial.

O primeiro desses eixos, o dos valores secular-racionais, envolve um conjunto de orientações que separam, sobretudo, as sociedades em que a religião é tomada como instituição social importante daquelas em que essa relevância é reduzida. Mas não é apenas na adesão religiosa que uma sociedade manifesta adesão a este tipo de orientações. A importância que os seus habitantes atribuem à família, o respeito às autoridades, a aceitação dos conflitos, a sua posição em relação a assuntos polêmicos, como aborto, eutanásia e suicídio, são também muito importantes na sua classificação (INGLEHART, 2001).

O segundo eixo organiza-se basicamente sobre a polarização entre valores materialistas e pósmaterialistas, que refletem uma mudança intergeracional de ênfase na segurança econômica e física para a ênfase na auto-expressão, bem-estar subjetivo e qualidade de vida (INGLEHART, 1990; 2001; 2002; INGLEHART \& WELZEL, 2005).

Esta mudança de direção seria comum a todas as sociedades de industrialização avançada nas quais as preocupações com a sobrevivência foram superadas e índices aceitáveis de bem-estar foram alcançados. Em tais sociedades, tem-se desenvolvido uma crescente preocupação com qualidade de vida, defesa do meio ambiente, bem como novas demandas por participação nos processos decisórios. Mas, assim como no caso das orientações seculares, outras questões também compõem esta dimensão. Os dados apresentados por Inglehart e Baker (2000) apontam que as sociedades que enfatizam valores de sobrevivência apresentam, dentre outras características, baixo bem-estar subjetivo, intolerância, baixo apoio à igualdade entre os sexos, níveis de confiança interpessoal reduzidos, pouco ativismo ambientalista e são relativamente favoráveis a formas de governo autoritárias. 
O mapa derivado do cruzamento destas duas dimensões, tomando como base os dados produzidos por seguidas ondas de surveys, contribui para a afirmação de que o desenvolvimento econômico favorece o estabelecimento de uma visão de mundo secularizada e pós-materialista. Países com renda per capita superior a 15.000 dólares anuais situam-se no topo das duas dimensões mencionadas acima, ou seja, são altamente secularizados e enfatizam de maneira significativa a auto-expressão (INGLEHART \& BAKER, 2000).

As zonas culturais de que tratamos anteriormente, entretanto, continuam nitidamente demarcadas quando são sobrepostas ao mapa. Os países que compartilham de uma determinada herança cultural aparecem agrupados quando está em questão sua adesão às orientações seculares e valores pós-materialistas. Esta mudança em curso, portanto, não ocorre de maneira linear e homogênea, mas orientada por trajetórias preestabelecidas pela configuração cultural de cada região.

Nesse processo de mudanças e permanências, os países latino-americanos representam um caso bastante interessante. A partir de uma comparação entre dados de 1981 e 1998, foram identificadas pequenas evidências de secularização entre os países em desenvolvimento da região, pois apenas Chile e México apresentaram evolução considerável nessa dimensão (ibidem).

No que diz respeito à segunda dimensão, entretanto, a situação é distinta, pois estas sociedades demonstraram uma tendência de movimentação em direção aos valores de auto-expressão.

Desse modo, ainda que a mudança cultural seja tímida no que diz respeito à secularização, existem indicações de que a trajetória em direção ao que podemos chamar de "pós-materialismo" verifica-se também nesta região do globo.

As conseqüências deste processo para o campo político são particularmente relevantes, pois, tal mudança nos valores culturais, em decorrência do processo de modernização e desenvolvimento econômico, relaciona-se de maneira significativa com as possibilidades de estabelecimento e desenvolvimento de sistemas políticos democráticos.

A melhoria dos indicadores socioeconômicos tem produzido uma série de condições favoráveis ao sucesso de formas de governo democráticas, como “[...] urbanização, educação em massa, especialização profissional, crescentes de redes organizacionais, maior igualdade de renda e uma diversidade de desenvolvimento associados que mobilizam a participação em massa na política" (INGLEHART, 2002, p. 147-148). No campo da cultura política, isto também ocorre, pois uma situação relativamente confortável no campo material tende "a aumentar a confiança interpessoal e a tolerância e leva à difusão de valores pós-materialistas que atribuem alta prioridade à auto-expressão e à participação no processo decisório” (ibidem).

Todas estas afirmações são sustentadas pelos autores a partir do cruzamento entre os dados do WVS de diferentes países e a pontuação que obtiveram em medidas de democratização formal e efetiva (INGLEHART \& WELZEL, 2005).

Este procedimento aponta que as sociedades enfatizadoras de valores de auto-expressão são aquelas que ocupam as melhores posições quando são verificados o respeito aos direitos políticos e às liberdades civis. A situação é inversa quando as sociedades manifestam mais adesão aos valores de sobrevivência.

Tratando da relação de causalidade entre variáveis culturais e os processos de democratização, os autores da teoria do desenvolvimento humano questionam a validade da tentadora tese de que a democracia é geradora de valores compatíveis com suas instituições. Por intermédio de evidências empíricas bastante fortes, Inglehart e Welzel (2005) demonstram que a direção causal que parte dos valores em direção às instituições é bem mais forte do que a contrária.

Respeitando o princípio da ordem temporal para a determinação de relações de causalidade entre variáveis, os autores valem-se de medidas de valores e de democracia coletadas em momentos distintos. O procedimento analítico adotado consiste em identificar o impacto produzido pelos processos de democratização ocorridos na década de 1980 sobre os valores de auto-expressão, medidos entre 1997 e 2002 e, na direção inversa, verificar o impacto de medidas de valores anteriores a 1990 sobre medidas de democracia coletadas entre 1997 e 2002. Os resultados obtidos com os modelos de regressão conduzem os analistas à refutação da tese de que o estabelecimento de instituições democráticas conduz à formação de uma cultura política congruente entre as massas (INGLEHART \& WELZEL, 2005). 
Tentando contribuir para o debate sobre este controverso tema no contexto específico da recente democratização brasileira, na próxima seção, apresentamos uma proposta de análise sobre o impacto do estabelecimento de instituições democráticas sobre os valores, crenças e atitudes políticas da população, a partir de uma comparação entre grupos etários distintos, submetidos a experiências de socialização distintas em seus períodos de mais suscetibilidade.

\section{PROBLEMA, DADOS E MÉTODO}

O processo de democratização brasileiro, amplamente discutido por inúmeros cientistas sociais, é repleto de peculiaridades que o tornam singular (DINIZ, 1985; MARTINS, 1986; STEPAN, 1989). Lenta e gradual, a transição levou 11 anos para restabelecer os direitos civis e mais cinco para que os cidadãos pudessem eleger seu candidato à presidência.

Podemos identificar ao menos três fases distintas nesse longo caminho: a primeira, que vai de 1974 a 1982, dominada totalmente pelos militares e com poucos avanços; a segunda, que vai de 1982 a 1985, também dominada pelos militares, mas com a incorporação de outros atores políticos que passam a desempenhar um importante papel; e, por fim, a fase que vai de 1985 a 1989, quando os militares deixam de desempenhar o papel principal, os civis assumem suas funções, e setores da sociedade civil organizada passam a participar do processo político (KINZO, 2001).

A partir desta periodização, podemos contabilizar aproximadamente duas décadas de democratização em curso e/ou vigência de instituições democráticas em nosso país. Ainda que seja um período muito curto para uma análise conclusiva envolvendo o tema da mudança de valores, é incontestável o fato de que já temos, ao menos, uma nova geração socializada sob a influência das instituições fundamentais de uma democracia liberal.

Partidos, parlamentos, eleições, direitos civis e políticos formais fazem parte da história de vida dos atuais adolescentes e jovens desde suas primeiras experiências de socialização política, que ocorreram nas décadas de 1980 e 1990.

A suposição de que tais experiências sob instituições democráticas tenham conduzido a uma cultura política democrática, entretanto, não pode ser automaticamente aceita. Como afirmam
Almond e Verba (1989), o processo de formação do padrão de atitudes e comportamentos de adultos em relação aos objetos políticos envolve um complexo de fatores.

Questionando afirmações de natureza psicológicas de que a formação deste padrão daria-se apenas nos primeiros anos da vida dos indivíduos, os autores argumentam que experiências na chamada "fase pré-adulta" e também na maturidade têm importância fundamental para a configuração das orientações políticas subjetivas. Isto não quer dizer que as experiências da primeira infância não tenham relevância, mas sim que "[...] early socialization experiences significantly affect an individual's basic personality predispositions and may therefore affect his political behavior, but numerous other factors intervene between these earliest experiences and later political behavior that greatly inhibit the impact of the former on the latter” (ALMOND \& VERBA, 1989, p. 266-267).

Começando na infância, mas estendendo-se por todas as demais fases da vida do indivíduo, devemos definir o processo de socialização política como “[...] o conjunto de experiências que, no processo de formação da identidade social do indivíduo, tem influência na cristalização do seu papel como ator significativo ou não no sistema político e as crenças que ele desenvolve em relação à política e as instituições [...]” (BAQUERO, 1997). Assim, dá-se continuamente, na medida em que as pessoas vivenciam suas experiências sociais em espaços distintos como a família e a escola, os seus padrões de atitudes e procedimentos vão sendo formados e/ou alterados (ALMOND \& POWELL JR., 1972).

Desta maneira, as experiências que ocorrem em instituições voltadas aos adultos ou pré-adultos, como sindicatos, clubes sociais e esportivos, ambientes de trabalho, dentre outros, são de fundamental importância para a formação da cultura política. Isto, entretanto, não impede-nos de apontar que as experiências de socialização que ocorrem na infância e na juventude são as que mais impactam as crenças, valores e atitudes políticos dos indivíduos (ROKEACH, 1968).

Acerca desta questão, as contribuições de Robert Dahl (1997) são de grande valia, pois o autor trata de afirmar a importância da socialização precoce diante dos demais momentos e experiências políticas. 
Segundo o autor, as crenças acerca dos objetos políticos são adquiridas nas primeiras duas décadas de vida de uma pessoa, período em que se é mais receptivo a elas. Quando este período é ultrapassado, os indivíduos tendem a possuir uma visão mais cristalizada, e suas crenças tendem a tornarem-se mais estáveis e menos suscetíveis a mudanças (idem).

Devemos concluir, portanto, que indivíduos brasileiros de faixas etárias distintas tiveram diferentes experiências de socialização política em decorrência da recente mudança institucional operada no país. Indivíduos que atualmente possuem de 15 a 25 anos viveram sob instituições democráticas desde seus nascimentos, ao passo que aqueles agrupados em faixas etárias mais elevadas passaram parte importante do seu período de mais suscetibilidade sob o domínio de um desenho institucional autoritário.

Não podemos deixar de considerar a hipótese de que estes últimos indivíduos, membros de gerações mais velhas, ao terem vivenciado as duas formas de governo, poderiam também ter suas orientações subjetivas alteradas positivamente pela democratização, passando a manifestar valores como tolerância, liberdade e pluralismo. Esse raciocínio, entretanto, não invalida o pressuposto aqui adotado de que, após as duas primeiras décadas de socialização, os valores e crenças tendem a estar mais consolidados, e as suas mudanças tendem a ser mais sutis (ROKEACH, 1968).

Assim, ainda que as mudanças ocorram em todos os grupos geracionais, é plausível supor que o efeito verificado nos mais jovens seja maior do que o encontrado nas demais. Ou seja, tomando como verdadeira a afirmação de que instituições democráticas favorecem o desenvolvimento de valores democráticos nos cidadãos que vivem sob sua influência, poderíamos supor a existência de diferenciais significativos nos valores e atitudes políticas entre diferentes grupos etários.

O fato de alguns desses grupos terem passado por experiências políticas distintas ao longo de suas vidas remete-nos também à interessante questão sobre as diferentes intensidades dos processos de socialização política em regimes democráticos e autoritários. Nestes últimos, devido à recorrente pretensão de controlar a vida social por meio da sua politização, mais atenção é dada à transmissão de valores à infância e à juventude, o que po- deria tornar os efeitos da socialização mais intensos nesse contexto político ${ }^{1}$.

Apesar de reconhecermos a pertinência de tal argumento e aceitarmos como pertinente a hipótese de que o processo de socialização política em democracias seja menos intenso do que em outras formas de governo, ele ainda ocorre, e seus efeitos devem ser diferentes entre os distintos grupos etários.

Para verificar a pertinência desta afirmação, propomos a análise de um conjunto de dados produzidos pela sondagem realizada entre 1995 e 1997 pelo projeto World Value Surveys, coordenado por Ronald Inglehart ${ }^{2}$.

Os procedimentos de análise aqui empregados consistem basicamente na aplicação de testes para identificar a associação entre alguns índices relacionados à cultura política e as idades dos entrevistados.

Os índices em questão são: 1) confiança institucional; 2) apoio à democracia; 3) preferência democracia-autocracia; 4) confiança interpessoal; 5) ativismo voluntário i; 6) ativismo voluntário ii; 7) pós-materialismo (aspirações por auto-expressão). Para a composição de cada um destes índices, inicialmente, foi realizada uma análise fatorial exploratória para identificar a pertinência da redução de um grupo de questões a uma única variável. Uma vez que tal análise tenha demonstrado a validade da redução de dimensionalidade, o índice foi construído com o somatório de valores encontrados em cada uma das questões pertinentes. As questões utilizadas para composição de cada um dos índices, bem como os procedimentos de recodificação empregados, serão apresentadas no momento em que a análise

1 Tal questionamento foi proposto pelo parecerista anônimo da Revista de Sociologia e Política, a quem agradecemos.

2 A base de dados utilizada é registrada com o n. 3975 no Inter-university Consortium for Political and Social Research. Como em nosso país não foi realizada a sondagem referente ao período de 1999-2002, a que se refere essa base, os dados da sondagem anterior (1995-1997) foram reproduzidos para fins de análises comparativas. A amostra representativa para o Brasil é composta por 1149 entrevistas. Os responsáveis pela distribuição dos dados para o país é o Centro de Estudos de Opinião Pública da Universidade Estadual de Campinas, a quem agradeço profundamente. 
debruçar-se sobre os temas correspondentes e detalhados em um apêndice, no fim do trabalho.

Como a idade dos entrevistados e os índices propostos não possuem uma distribuição normal, ao invés de utilizarmos o coeficiente de correlação de Pearson, empregaremos o teste nãoparamétrico de correlação de Spearman para identificar a existência e a natureza (positiva ou negativa) da relação entre as variáveis.

Antes de iniciarmos a análise propriamente dita, consideramos que seja interessante apresentar uma tabela em que as diferentes idades dos entrevistados aparecem agrupadas com seus respectivos períodos de maior receptividade para a formação de seus valores, crenças e atitudes políticas.

TABELA 1 - GRUPOS ETÁRIOS E PERÍODOS DE MAIOR RECEPTIVIDADE

\begin{tabular}{|c|c|c|}
\hline \multicolumn{2}{|c|}{$\begin{array}{l}\text { GRUPOS ETÁRIOS EM } \\
1995 / 1997^{\star 4}\end{array}$} & \multirow{2}{*}{$\begin{array}{c}\begin{array}{c}\text { PERÍODO DE } \\
\text { MAIOR } \\
\text { RECEPTIVID ADE } \\
\text { 1995/97 ** }\end{array} \\
1980 / 82 \text { a } 2000 / 05\end{array}$} \\
\hline \multirow[t]{2}{*}{15 a 20 anos } & 15 & \\
\hline & 20 & $1975 / 76$ a $1994 / 96$ \\
\hline \multirow[t]{2}{*}{21 a 25 anos } & 21 & $1974 / 76$ a $1994 / 96$ \\
\hline & 25 & $1970 / 72$ a $1990 / 02$ \\
\hline \multirow[t]{2}{*}{26 a 30 anos } & 26 & $1969 / 71$ a $1989 / 91$ \\
\hline & 30 & $1965 / 67$ a $1985 / 87$ \\
\hline \multirow[t]{2}{*}{31 a 35 anos } & 31 & $1964 / 66$ a $1984 / 86$ \\
\hline & 35 & $1960 / 62$ a $1980 / 82$ \\
\hline \multirow[t]{2}{*}{36 a 40 anos } & 36 & $1959 / 61$ a $1979 / 81$ \\
\hline & 40 & $1955 / 57$ a $1975 / 77$ \\
\hline \multirow[t]{2}{*}{41 a 45 anos } & 41 & $1954 / 56$ a $1974 / 76$ \\
\hline & 45 & $1950 / 52$ a $1970 / 72$ \\
\hline \multirow[t]{2}{*}{46 a 50 anos } & 46 & $1949 / 51$ a $1969 / 71$ \\
\hline & 50 & $1945 / 47$ a $1965 / 67$ \\
\hline
\end{tabular}

NOTAS:

1. * O período de maior receptividade vai do nascimento aos 20 anos, ou seja, as duas primeiras décadas de vida dos indivíduos.

2. ** Os dados foram coletados entre 1995 e 1997, de modo que as idades e períodos de receptividade variam dentro desse intervalo.

Podemos identificar que os três primeiros grupos foram os que tiveram a maior parte dos seus respectivos períodos de receptividade sob a vigência das instituições democráticas ou durante o processo de transição para aquelas. Apesar de os períodos em questão serem, evidentemente, curtos demais para a verificação de mudanças nos valores de uma população, como propomos uma análise individual, o enfoque sobre as duas primeiras décadas de receptividade parece-nos adequado. Trata-se, portanto, de verificar se a experiência individual de viver esse período de socialização sob a influencia de um processo de democratização ou de instituições democráticas instaladas produziu algum impacto sobre os valores políticos dos entrevistados.

\section{COMPONENTES DA CULTURA POLÍTICA E EXPERIÊNCIA DEMOCRÁTICA}

O percurso de análise que iremos adotar nesta seção corresponde à divisão dos estudos sobre cultura política em três grandes grupos, como proposta por Inglehart e Welzel (2005) e apresentada na seção inicial deste artigo.

Assim, iniciamos pelo conjunto de variáveis enfatizadas pela chamada "perspectiva da legitimidade”. Como já destacamos, tal abordagem entende que todos os sistemas políticos, mas principalmente as democracias, precisam gozar de legitimidade entre as massas. Confiança nas instituições e o suporte a organizações de caráter democrático seriam variáveis fundamentais para a explicação do processo de implantação e consolidação destes sistemas (MISHLER \& ROSE, 2001; SELIGSON, 2002).

A discussão sobre o impacto da confiança institucional ou do suporte democrático para o funcionamento da democracia ultrapassa os limites do problema que propomos neste trabalho. Desta maneira, ainda que pesquisas empíricas recentes indiquem uma baixa correlação entre índices de confiança institucional e medidas de democracia formal e efetiva (INGLEHART \& WELZEL, 2005), acreditamos que, diante dos objetivos que propomos, seja interessante lançar nosso olhar sobre o tema.

Como já indicamos na exposição dos procedimentos metodológicos empregados, inicialmente, realizamos uma análise fatorial exploratória para identificar a viabilidade da construção de um índice de confiança institucional, envolvendo a confiança no congresso nacional, no governo e nos partidos políticos. 
O resultado deste procedimento foi bastante positivo, sendo extraído um fator que explica $73,28 \%$ da variação (Tabela 2), o que ampara a redução das três variáveis a um único índice de Confiança Institucional.

TABELA2-ANÁLISE FATORIAL COM VARIÁVEIS DE CONFIANÇA INSTITUCIONAL

\begin{tabular}{l|c|}
\hline VARIÁVEL & COMPOIIIITE \\
\hline Con fiança no Congresso Nacional & 0,875 \\
Confiança no governo & 0,824 \\
P artidos políticos & 0,869 \\
\% variaçẫo & 73,28 \\
\hline
\end{tabular}

NOTA: Método de extração: análise de componentes principais.

As variáveis, que originalmente tinham como opções a escala "muito/razoavelmente/pouco/não confia", foram recodificadas da seguinte forma: 0 para as opções "pouco/não confia” e 1 para "muito/ razoavelmente". Desta forma, o índice somatório de confiança institucional proposto vai de 0 a 3 .

Confirmando tendência já identificada no início da década de 1990 por pesquisa nacional (MOISÉS, 1995), os dados aqui analisados indicam que $44,3 \%$ da população não deposita qualquer confiança nas instituições destacadas, pois situam-se no ponto 0 do índice.

Remetendo-nos ao tema central deste trabalho, verificamos que existe uma correlação significativa $(0,000)$ entre este índice e a idade dos indivíduos, entretanto, ela é positiva e muito baixa $(0,118)$, o que indica que a confiança institucional tende a aumentar de maneira muito sutil quando a idade também aumenta. Desta forma, somos levados a concluir que a experiência de socialização sob a transição ou vigência da institucionalidade democrática não tende a afetar positivamente a confiança depositada pelos entrevistados em relação às instituições desta forma de governo.

Essas evidências, todavia, não contribuem para negar as teses institucionais, pois estes baixos níveis de confiança, entre as faixas etárias mais jovens, podem ser explicados pelo deficiente desempenho das instituições em questão, pois a con- fiança pode ser entendida como resultante da compatibilidade entre o desempenho institucional e as justificativas normativas para o seu estabelecimento e manutenção (MOISÉS, 2005).

No caso brasileiro, a incapacidade das instituições democráticas garantirem melhores condições de existência para a população, bem como os constantes episódios de corrupção e uso indevido da estrutura estatal, pode estar conduzindo as novas gerações, nascidas no período de sua implantação ou após o seu estabelecimento, a esta postura de desconfiança.

O segundo tema destacado pela abordagem da legitimidade diz respeito ao apoio à democracia, através de um índice construído a partir do grau de concordância dos entrevistados com a seguinte afirmação: "A democracia pode ter seus problemas, mas ainda é a melhor forma de governo". As opções aqui são "discorda muito/discorda/concorda/concorda muito”, gerando um índice que vai de 0 a 3.

A correlação encontrada aqui também é significativa $(0,000)$, positiva e fraca $(0,118)$, indicando que o aumento na variável idade tende a ser acompanhado de um aumento muito sutil do apoio à democracia. Novamente, não pode ser sustentada a afirmação de que a experiência democrática nos períodos de mais receptividade das novas gerações tenha produzido apoio maior à democracia.

Complementarmente a este suporte, verificamos também a preferência da população entre democracia e autocracia para identificar de maneira mais precisa em que medida o grau de legitimidade democrática varia em relação à idade dos indivíduos. Assim como no caso da confiança institucional, construímos um índice de preferência democracia-autocracia com as variáveis obtidas pela posição dos indivíduos em relação as seguintes afirmações relacionadas a diferentes formas de governar: 1) tendo um líder forte que não incomode-se com congresso/senado e eleições; 2) tendo um governo militar; 3) tendo um sistema político democrático (Tabela 3).

Nos três itens, a escala de opções é "muito bom/bom/mal/muito mal”. Como o sentido das duas primeiras questões é invertido na terceira, os valores atribuídos às respostas também foram invertidos. Portanto, o índice vai de 0 a 9. 


$\begin{gathered}\text { TABELA3-ANÁLISE FATORIAL COM VARIÁVEIS DE } \\
\text { PREFERENNCIA } \\
\text { AUTOCRACIA }\end{gathered}$
\begin{tabular}{|l|c|}
\hline \multicolumn{1}{|c}{ DEMOCRACIA- } \\
\hline VARIÁVEL & COMPONENTE \\
\hline Liderança forte & 0,706 \\
Governo militar & 0,805 \\
Sistema político democrático & 0,481 \\
\% variaçẫo & 45,9 \\
\hline
\end{tabular}

FONTE: World Values Surveys (1999-2002).

NOTA: Método de extração: análise de componentes principais

Nesse caso, o teste demonstrou não haver associação significativa $($ sig. $=0,18)$ entre as variáveis, o que equivale a dizer que a preferência entre democracia e autocracia não está relacionada com a idade dos indivíduos, ao menos no contexto brasileiro.

A partir do exposto, podemos afirmar que não existem correlações expressivas, como os valores abaixo resumem (Tabela 4), entre as variáveis enfatizadas pela abordagem da legitimidade e as idades dos entrevistados, o que colabora a interpretação de que as experiências de socialização política dos indivíduos mais jovens, que viveram seus momentos de receptividade maior no período de transição ou de vigência da democracia, não produziram efeitos verificáveis no sentido do estabelecimento de uma postura mais congruente com a consolidação e aprofundamento deste sistema político.

TABELA 4 - CORRELAÇÕES ENTRE ÍNDICES E IDADE: ABORDAGEM DA LEGITIMIDADE

\begin{tabular}{|l|c|}
\hline ÍNDICE & ID ADE \\
\hline Confiança institucional & $0,118^{*}$ \\
Apoio à democracia & $0,118^{*}$ \\
Preferência democracia-autocracia & - \\
\hline
\end{tabular}

FONTE: World Values Surveys (1999-2002). NOTAS:

1. * Nível de significância de 0,00

2. ** Não existe associação significativa (sig.=0).

A partir deste momento, pretendemos voltar nossa atenção para as variáveis que são enfatizadas pela segunda abordagem culturalista que apresen- tamos na seção anterior: a comunitarista.

Essa abordagem caracteriza-se pela preocupação com valores que extrapolam a dimensão institucional da política e que ligam os cidadãos à vida pública diária, bem como fortalecem seus compromissos e lealdades com sua comunidade. Tais orientações subjetivas comunitárias levariam ao desenvolvimento de capital social que, por sua vez, refletir-se-ia no ativismo em associações voluntárias e também em confiança interpessoal (PUTNAM, 1996, 2000).

É a associação entre essas variáveis, definidas como relevantes para o desempenho democrático, e as idades dos indivíduos que compõe a amostra que iremos agora analisar.

A primeira delas seria a participação em associações voluntárias. Por meio de análise fatorial exploratória, identificamos que as participações em organizações religiosas, artístico-educacionais, ambientais e de caridade podem ser agrupadas para formar um índice somatório de Ativismo Voluntário I (Tabela 5), que varia de 0 a 4 e que refere-se ao que podemos chamar de "organizações sociais".

TABELA5 -ANÁLISE FATORIAL COM VARIÁVEIS DE ATIVISMO VOLUNTÁRIO

\begin{tabular}{|l|c|}
\hline VARIÁVEL & COMPONENTE \\
\hline Religiosas & 0,674 \\
Artísticas e educacionais & 0,553 \\
Ambientais & 0,550 \\
Caridade & 0,720 \\
\% Variaçẫo & 39,5 \\
\hline
\end{tabular}

FONTE: World Values Surveys (1999-2002).

NOTA: Método de extração: análise de componentes principais.

Nesse item, encontramos uma correlação significativa $(0,000)$, positiva e baixa $(0,120)$, o que indica a existência de uma associação muito sutil entre a idade e a participação nestas instituições. Assim, como verificamos anteriormente a respeito das variáveis relacionadas ao tema da legitimidade, aqui também as faixas etárias mais jovens não demonstram uma postura mais cívica. Apesar de a associação ser discreta, a elevação na idade tende a ser acompanhada de aumento no ativismo. 
Também utilizamos uma medida de ativismo em instituições mais essencialmente relacionadas ao ambiente político, por meio de análise fatorial, envolvendo a participação voluntária em sindicatos, partidos e organizações profissionais (Tabela 6) e encontramos uma correlação não significativa (sig. $=0,143$ ), demonstrando que este tipo de ativismo não está associado à variável idade na amostra analisada.

TABELA6 - ANÁLISE FATORIAL COM VARIÁVEIS DE ATIVISMO VOLUNTÁRIO II

\begin{tabular}{|l|c|}
\hline VARIÁVEL & COMPONENTE \\
\hline Sindicatos & 0,795 \\
Partidos & 0,479 \\
Organizaçốes profissionais & 0,776 \\
\% variaçăo & 48,8 \\
\hline
\end{tabular}

FONTE: World Values Surveys (1999-2002).

NOTA: Método de extração: análise de componentes principais.

No que diz respeito à atuação voluntária dos cidadãos em ambos os grupos de instituições, verificamos que a experiência democrática nos períodos de receptividade maior das faixas etárias mais jovens não conduziu a um aumento na disposição de participar.

Uma última variável destacada pela abordagem comunitarista é a confiança interpessoal, apontada como importante componente de uma configuração sociocultural que favorece tanto o estabelecimento quanto a estabilidade de sistemas democráticos (ALMOND \& VERBA, 1989; PUTNAM, 1996).

Nesse caso, valemo-nos da seguinte questão: "Você diria que podemos confiar na maioria das pessoas ou que sempre devemos tomar cuidado no tratamento com as pessoas?” A variável binária gerada pelas respostas compõe um índice de Confiança Interpessoal que varia de 0 a 1 . $O$ teste estatístico demonstrou que não existe uma associação significativa entre esta variável e a idade dos entrevistados $(0,13$ com sig. $=0,653)$.

A síntese das correlações entre as variáveis comunitárias e a idade (Tabela 7), corrobora a afirmação de que a experiência de socialização política na vigência de instituições democráticas dos cidadãos de faixas etárias mais jovens ainda não produziu efeitos positivos constatáveis.
TABELA 7 - CORRELAÇÕES ENTRE ÍNDICES E IDADE: ABORDAGEM COMUNITARISTA

\begin{tabular}{|l|c|}
\hline ÍNDICE & IDADE \\
\hline Ativismo voluntário I & $0,120^{*}$ \\
Ativismo voluntário II & $-*$ \\
Confiança interpessoal & $-* *$ \\
\hline
\end{tabular}

FONTE: World Values Surveys (1999-2002). NOTAS:

1. * Nível de significância de 0,00.

2. ** Não existe associação significativa (sig. $=0,143$ ).

3. *** Não existe associação significativa (sig. $=0,653$ )

Por fim, trataremos agora da abordagem que tem sido chamada de "teoria do desenvolvimento humano". Como discutimos na primeira parte deste artigo, os defensores desta explicação associam o desenvolvimento socioeconômico a um conjunto de condições favoráveis à democracia (INGLEHART, 2002). No campo dos valores, este desenvolvimento contribuiria para o incremento na confiança interpessoal, tolerância e para o estabelecimento de valores pós-materialistas que atribuem prioridade à auto-expressão e à participação no processo decisório.

Ainda que em trabalho recente Inglehart e Welzel (2005) tenham apresentado evidências suficientes para afirmar que a direção causal partindo dos valores pós-materialistas em direção às instituições é bem mais forte do que a contrária, acreditamos que a inclusão desta abordagem em nossos testes seja interessante.

Assim, tratamos de medir a associação entre os valores pós-materialistas de auto-expressão e a idade dos entrevistados para verificar a existência de diferenciais significativos entre as faixas etárias que tiveram diferentes experiências de socialização política. Para tanto, nos valemos do índice de pós-materialismo composto por 12 itens elaborado por Inglehart.

Os testes indicaram que a correlação entre as variáveis é significativa $(0,001)$, fraca e negativa $(-0,101)$, ou seja, a redução da idade tende a ser acompanhada de um sutil aumento no índice de pós-materialismo. É importante notar que nas análises aqui realizadas essa é a primeira vez que uma associação negativa aparece, ainda que seja reduzida. 
Apesar de o Brasil fazer parte do grupo de países em desenvolvimento, portanto, não apresentar as condições para a generalização da chamada "síndrome de pós-materialismo", identificada nas nações desenvolvidas (INGLEHART, 1997; 2001), podemos notar um início tímido de mudança intergeracional.

Esta associação, entretanto, não pode ser explicada como efeito da experiência das camadas mais jovens sob as instituições democráticas. Fortes evidências empíricas validadas mundialmente apontam que, em uma rigorosa análise de causalidade, o impacto dos valores que compõem a síndrome de pós-materialismo sobre os processos de democratização é bem maior do que o inverso. Entretanto, a conseqüência mais importante destes resultados diz respeito a um paradoxo que precisa ser analisado em um nível de profundidade e sofisticação que este artigo não pretende ter. O paradoxo diz respeito à convivência de uma tendência (fraca) de adesão entre as camadas mais jovens a valores caracterizados como pós-materialistas sem que exista uma correspondente adesão a outros indicadores de uma cultura política favorável à democracia.

É claro que a persistência deste fenômeno é incerta, uma vez que a mudança intergeracional, em termos de pós-materialismo no Brasil, ainda é uma tendência muito sutil, quase insignificante estatisticamente. Se tal situação continuará ou não a ocorrer e quais serão seus efeitos sobre o futuro de nossa democracia são perguntas que só poderão ser respondidas quando sondagens subseqüentes forem realizadas e os seus dados forem analisados em perspectiva longitudinal.

\section{CONCLUSÕES}

Como a análise precedente aponta, com exce- ção do índice de pós-materialismo, nenhuma das variáveis enfatizadas por abordagens da chamada "teoria da cultura política” estão associadas de maneira considerável com a idade dos entrevistados no sentido negativo, o que poderia indicar uma cultura política mais congruente com a forma democrática de governo entre faixas etárias mais jovens. Como verificamos, o contrário ocorreu na maioria das vezes, pois as associações conduziram à conclusão de que confiança institucional, apoio à democracia, preferência democracia-autocracia, dentre outras medidas, tenderam à elevação com o avançar da idade dos indivíduos.

Tais resultados não pretendem conduzir-nos a conclusões contundentes sobre o problema da relação causal entre valores e instituições para o caso brasileiro. Pretensão desta ordem careceria de um período maior de vigência democrática em nosso país e também de séries de dados mais longas.

Longe disto, este estudo limitou-se a apresentar indicações de que existe uma juventude nascida ou socializada na vigência de instituições democráticas que não demonstra possuir valores políticos significativamente diferentes de outros estratos da população que tiveram outras experiências socializadoras.

Ainda que nossa democracia possua uma história muito recente e os dados não possibilitem uma análise comparativa ao longo do tempo para a identificação de tendências consistentes, acreditamos que a análise aqui proposta seja um ponto de partida relevante para a identificação de tais problemas. Estes, por sua vez, são da mais alta relevância para um projeto de consolidação e aprofundamento da democracia nacional.

Ednaldo Aparecido Ribeiro (ednaldorip@uol.com.br) é doutorando em Sociologia na Universidade Federal do Paraná (UFPR) e Professor de Ciências Sociais na Universidade Estadual de Maringá (UEM).

\section{REFERÊNCIAS BIBLIOGRÁFICAS}

ALMOND, G. 1989. The Intellectual History of the Civic Culture Concept. In : ALMOND, G. \& VERBA, S. (eds.). The Civic Culture Revisited. London : Sage.

ALMOND, G. \& POWELL JR., B. 1972. Uma teoria da política comparada. Rio de Janeiro : Zahar.

ALMOND, G. \& VERBA, S. 1989. The Civic Culture : Political Attitudes and Democracy in Five Nations. New York : Sage. 
BAQUERO, M. 1997. O papel dos adolescentes no processo de construção democrática no Brasil : um estudo preliminar de socialização política. Cadernos de Ciência Política, Porto Alegre, n. 8, p. 3-34, jul.

BELL, D. 1977. O advento da sociedade pósindustrial. São Paulo : Cultrix.

CARVALHO, J. M. 1999. O motivo edênico no imaginário social brasileiro. Revista Brasileira de Ciências Sociais, Rio de Janeiro, v. 13, n. 38, p. 63-79, out.

DAHL, R. 1997. Poliarquia. São Paulo : USP.

DALTON, R. J. 2000. Value Change and Democracy. In : PHARR, S. J. \& PUTNAM, R. D. (eds.). Disaffected Democracies : What's Troubling the Trilateral Countries? Princeton : Princeton University.

DINIZ, E. 1985. A transição política no Brasil : uma reavaliação da dinâmica da abertura. $D a-$ dos, Rio de Janeiro, v. 28, n. 3, p. 329-346, out.

FUKUYAMA, F. 1995. Trust: The Social Virtues and the Creation of Prosperity. New York : Free.

HUNTINGTON, S. 1997. O choque de civilizações e a recomposição da ordem mundial. Rio de Janeiro : Objetiva.

INGLEHART, R. 1988. The Renaissance of Political Culture. American Political Science Review, Washington, D. C., v. 82, n. 4, p. 12031229, Dec.

1990. Cultural Shift in Advanced Industrial Society. Princeton : Princeton University.

2001. Modernización y posmodernización : el cambio cultural, económico y político en 43 sociedades. Madrid : Centro de Investigaciones Sociológicas.

2002. Cultura e democracia. In : HARRINSON, L. E. \& HUNTINGTON, S. A cultura importa. Rio de Janeiro : Record.

INGLEHART, R. \& BAKER, W. E. 2000. Modernization, Cultural Change and the Persistence of Tradition : Empirical Evidence from 65 Societies. American Sociological Review, Philadelphia, v. 65, p. 19-51, Feb.
INGLEHART, R. \& WELZEL, C. 2005. Modernization, Cultural Change, and Democracy: The Human Development Sequence. Cambridge : Cambridge University.

KARL, T. L. \& SCHMITTER, P. C. 1991. Modes of Transition in Latin America, Southern and Eastern Europe. International Social Science Journal, n. 128, p. 269-284, May.

KINZO, M. D. 2001. A democratização brasileira : um balanço do processo político desde a transição. São Paulo em Perspectiva, São Paulo, v. 15, n. 4, p. 3-12, out.-dez.

KLINGEMANN, H. \& FUCHS, D. (eds.). 1995. Citizens and the State. Collec. Beliefs in government, v. 1. Oxford : Oxford University.

LAMOUNIER, B. 1991. Democracia e reforma institucional no Brasil : uma cultura política em mudança. Dados, Rio de Janeiro, v. 34, n. 3, p. 311-342, out.

MARTINS, L. 1986. The Liberalization of the Authoritarian Rule in Brazil. In : O’DONNELL, G.; SCHMITTER, P. C. \& WHITEHEAD, L. (eds.). Transitions from Authoritarian Rule. Baltimore : Johns Hopkins University.

MISHLER, W. \& ROSE, R. 2001. Political Support for Incomplete Democracies : Realist vs. Idealist Theories and Measures. International Political Science Review, Washington, D. C., v. 22, n. 4, p. 303-320, Dec.

MOISÉS, J. A. 1995. Os brasileiros e a democracia : bases sócio-políticas da legitimidade democrática. São Paulo : Ática.

2005. A desconfiança nas instituições democráticas. Opinião Pública, Campinas, v. 11, n. 1, p. 33-63, mar.

MULLER, E. N. \& SELIGSON, M. A. 1994. Civic Culture and Democracy : The Question of Causal Relationships. American Political Science Review, Washington, D. C., v. 88, n. 3, p. 635-652, Sept.

NEWTON, K. \& NORRIS, P. 2000. Confidence in Public Institutions : Faith, Culture, or Performance? In : PHARR, S. \& PUTNAM, R. (eds.). Disaffected Democracies : What's Troubling the Trilateral Countries? Princeton : Princeton University. 
NORRIS, P. 2002. Democratic Phoenix : Political Activism Worldwide. Cambridge : Cambridge University.

O'DONNELL, G. \& SCHMITTER, P. C. 1986. Tentative Conclusions about Uncertain Democracies. In : O’DONNELL, G.; SCHMITTER, P. C. \& WHITEHEAD, L. (eds.). Transitions from Authoritarian Rule. Baltimore : Johns Hopkins University.

PHARR, S. \& PUTNAM, R. (eds.). 2000. Disaffected Democracies : What's Troubling the Trilateral Countries? Princeton : Princeton University.

PRZEWORSKI, A. 1992. The Games of Transition. In : MAINWARING, S.; O’DONNELL, G. \& VALENZUELA, A. (eds.). Issues in Democratic Consolidation: The New South American Democracies in Comparative Perspective. Notre Dame : University of Notre Dame.

PUTNAM, R. 1996. Comunidade e democracia : a experiência da Itália moderna. Rio de Janei- ro : Fundação Getúlio Vargas.

2000. Bowling Alone : The Collapse and Revival of American Community. New York : Simon and Schuster.

RENNÓ, L. 1998. Teoria da cultura política : vícios e virtudes. BIB, Rio de Janeiro, n. 45, p. 71-91, mar.

ROKEACH, M. 1968. Beliefs, Attitudes and Values. San Francisco : Jossey-Bass.

SALES, T. 1994. Raízes da desigualdade social na cultura política brasileira. Revista Brasileira de Ciências Sociais, Rio de Janeiro, n. 25, p. 26-37, jul.

SELIGSON, M. 2002. The Renaissance of Political Culture or the Renaissance of the Ecological Fallacy. Comparative Politics, New York, n. 2, p. 281-293.

STEPAN, A. (ed.). 1989. Democratizando o Brasil. Rio de Janeiro : Paz e Terra.

WEBER, M. 1996. A ética protestante e o espírito do capitalismo. São Paulo : Pioneira.

\section{OUTRAFONTE}

WORLD VALUES SURVEYS. 1999-2002. Disponível em: http:// www.worldvaluessurvey.org/. Acesso em: 3.maio.2007.

\section{APÊNDICE}

Fontes de dados utilizadas na composição dos índices e respectivos procedimentos de recodificação.

\section{CONFIANÇA INSTITUCIONAL}

Índice que varia de 0 a 3, composto pelas respostas às variáveis V155 (Confiança no parlamento/Congresso Nacional), V153 (Confiança no governo) e V154 (Confiança nos partidos políticos) do WVS 1999-2002 ICPSR 3975. As variáveis, que originalmente eram compostas pela escala "muito=1/razoavelmente $=2 /$ pouco=3/não confia $=4$ ", foram recodificadas como binárias: "pouco/não confia=0" e "muito/ razoavelmente= 1 ".

\section{APOIO À DEMOCRACIA}

Varia de 0 a 3, sendo composto pelas respostas à V172 do WVS 1999-2002 - ICPSR 3975. A variável originalmente era composta por uma escala de concordância com a afirmação, “A democracia pode ter seus problemas, mas ainda é a melhor forma de governo", apresentando as opções "concordo muito $=1 /$ Concordo $=2 /$ Discordo=3/Discordo Muito=4”. A recodificação realizada inverteu a escala como segue: "Discorda muito=0/Discorda=1/Concorda=2/Concorda Muito=3” 


\section{PREFERÊNCIA POR DEMOCRACIA-AUTO- CRACIA}

Índice que varia de 0 a 9, composto pelas variáveis V164, V166 e V1671 do WVS 1999-2002 - ICPSR 3975, sobre as avaliações dos entrevistados em relação a três situações particulares: 1) tendo um líder forte que não incomode-se com congresso/senado e eleições; 2) tendo um governo militar; 3) tendo um sistema político democrático. Originalmente, as opções eram "muito bom=1/bom=2/mal=3/muito mal=4". No caso das duas primeiras questões, que possuem sentido negativo em relação à postura democrática, as respostas foram recodificadas da seguinte maneira: "muito bom $=0 /$ bom $=1 / \mathrm{mal}=2 /$ muito $\mathrm{mal}=3$ ". Na terceira, cujo sentido é positivo, a recodificação foi realizada como segue: "muito bom=3/ bom $=2 /$ mal=1/muito mal=0".

\section{CONFIANÇA INTERPESSOAL}

Índice binário ( 0 ou 1 ) obtido por meio da variável V025 do WVS 1999-2002 - ICPSR 3975. Originalmente, comportava as opções “A maioria das pessoas são confiáveis=1/Precisamos tomar cuidado no tratamento com as pessoas=2”. Configuração após a recodificação: “A maioria das pessoas são confiáveis=1/Precisamos tomar cuidado no tratamento com as pessoas $=0$ ".

\section{ATIVISMO VOLUNTÁRIO I}

Varia de 0 a 4, composto pelas respostas às questões V028, V030, V033, V035, acerca da participação voluntária em organizações de caráter social. As questões originalmente apresentavam as opções "membro ativo $=1 /$ membro inativo=2/não participa=3”. Tornando-as binárias, a recodificação realizada foi a seguinte: "membro ativo /membro inativo=1/não participa $=0 "$.

\section{ATIVISMO VOLUNTÁRIO II}

Varia de 0 a 3 , composto pelas respostas às questões V031, V032, V034, acerca da participação voluntária em organizações de caráter político. As questões, originalmente, apresentavam as opções "membro ativo=1/membro inativo=2/não participa=3”. Tornando-as binárias, a recodificação realizada foi a seguinte: "membro ativo /membro inativo=1/não participa $=0 "$.

7. PÓS-MATERIALISMO (ASPIRAÇÕES POR AUTO-EXPRESSÃO)

Índice que varia de 0 a 5 (escala que parte do $0=$ materialista e vai até 05 = pós-materialista), já computado na base de dados do WVS 19992002 - ICPSR 3975 sob a denominação de Y001. 
POLITICAL CULTURE, INSTITUTIONS AND DEMOCRATIC EXPERIENCE IN BRAZIL

Ednaldo Aparecido Ribeiro

Through analysis of data produced by the World Values Surveys project, this paper attempts to identify the impact that the period in which democratic institutions have come to prevail has had on the younger sectors of the population, precisely those who have had their political socialization during this period. Beginning from the premise that the incorporation of political values is rooted primarily in the first two decades of an individual's life, we seek to verify the validity of the hypothesis that democratic institutions tend to favor the establishment of values that are consistent with and amenable to their consolidation and deepening. For these purposes, we have tested the association between the variable "age" and some measures of values and political attitudes that have been emphasized by different approaches to what has been called "the theory of political culture". The results obtained run counter to the aforementioned thesis, or do so at least in the Brazilian case, insofar as values that are significantly different according to age group have not been identified for those who underwent their political socialization under historically different institutional arrangements.

KEYWORDS: democratic institutions; political values; political socialization; age groups. 
CULTURE POLITIQUE, INSTITUTIONS ET EXPÉRIENCE DÉMOCRATIQUE AU BRÉSIL Ednaldo Aparecido Ribeiro

Cet article cherche à identifier par l'intermédiaire de données issues du projet World Values Surveys, l'effet que la période où étaient en vigueur les institutions démocratiques dans notre pays a eu sur les tranches d'âge plus jeunes de la population, lors de leurs expériences de socialisation politique. En nous appuyant sur l'idée que l'incorporation de valeurs politiques se produit surtout au cours de deux premières décennies de la vie de l'individu, nous avons cherché à vérifier la valeur de l'hypothèse selon laquelle les institutions démocratiques ont tendance à favoriser la mise en place de valeurs congruentes et favorables à sa consolidation et à son enracinement. A cet effet, nous avons fait appel à des testes d'association entre la variante âge et certaines mesures de valeurs et comportements politiques qui sont mis en relief par des approches de la «théorie de la culture politique». Les résultats obtenus s'opposent à la thèse ci-dessus, au moins dans le cas brésilien, puisque des valeurs et des comportements nettement différents entre les tranches d'âge ayant leur procès de socialisation politique sous plusieurs compositions institutionnelles n’ont pas été identifiés.

MOTS-CLÉS: institutions démocratiques; valeurs politiques; socialisation politique; tranches d’âge. 